\title{
Supporting Information: \\ Dominant Role of Quantum Anharmonicity in the Stability and Optical Properties of Infinite Linear Acetylenic Carbon Chains
}

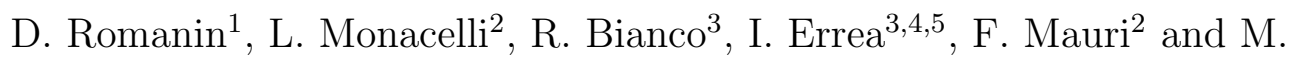
Calandra ${ }^{1,6}$

${ }^{1}$ Sorbonne Université, CNRS, Institut des Nanosciences de Paris, UMR7588, F-75252, Paris, France

${ }^{2}$ Dipartimento di Fisica, Universitá di Roma La Sapienza, Piazzale Aldo Moro 5, I-00185 Roma, Italy

${ }^{3}$ Centro de Física de Materiales (CSIC-UPV/EHU), Manuel de Lardizabal pasealekua 5, 20018 Donostia-San Sebastián, Basque Country, Spain

${ }^{4}$ Fisika Aplikatua 1 Saila, Gipuzkoako Ingeniaritza Eskola, University of the Basque Country (UPV/EHU), Europa Plaza 1, 20018, Donostia San

Sebastián, Basque Country, Spain

${ }^{5}$ Donostia International Physics Center (DIPC), Manuel de Lardizabal pasealekua 4, 20018 Donostia San Sebastián, Basque Country, Spain

${ }^{6}$ Department of Physics, University of Trento, Via Sommarive 14, 38123 Povo, Italy

October 11, 2021 


\section{Contents}

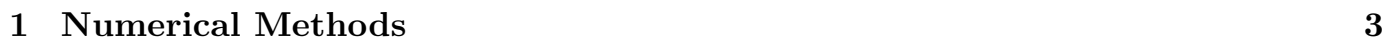

\begin{tabular}{|lll}
2 & Vibrational Properties & $\mathbf{5}$
\end{tabular}

2.1 Different contributions to the temperature dependent phonon frequency . . . 5

2.2 Static anharmonic phonon dispersion of carbyne $\ldots \ldots \ldots . \ldots 9$

2.3 Dynamical anharmonic phonon dispersion of carbyne . . . . . . . . . . . . . . 11

2.4 Temperature stability of the metallic phase $\ldots \ldots \ldots \ldots$. . . . . . . 13

2.5 Extrapolation of the charge density wave critical temperature from free energy

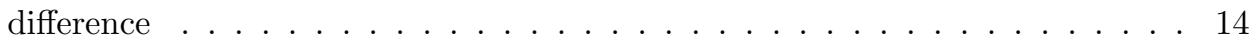

$2.6 \quad$ Quantum anharmonic relaxation of polyyne's lattice parameter . . . . . . . . 15

$\begin{array}{lll}3 & \text { Electronic Properties } & 16\end{array}$

3.1 Self-consistent $G W$ electronic bandstructure of polyyne at the PBE0 equilibrium geometry $\ldots \ldots \ldots \ldots \ldots \ldots \ldots$

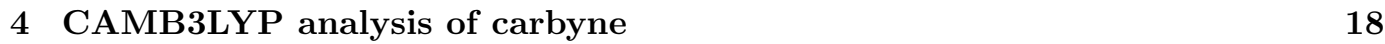




\section{Numerical Methods}

Density functional theory calculations are performed both with the plane-wave pseudopotential method, using the Perdew-Burke-Ernzerhof (PBE) exchange-correlation (xc) functional and Quantum ESPRESSO [1, 2], and with localized gaussian basis, using both the PBE0 and CAMB3LYP hybrid xc functionals and CRYSTAL [3, 4. In the Quantum ESPRESSO code we use a norm-conserving pseudopotential with the kinetic energy cut-off set to $65 \mathrm{Ry}$, while that for the density is set to $650 \mathrm{Ry}$, and a threshold of $10^{-9}$ Ry on the total energy. In the CRYSTAL code we use the triple- $\xi$-polarized Gaussian type basis set [5] with real space integration tolerances of 7-7-7-15-30 and an energy tolerance of $10^{-10}$ Ha for the total energy convergence.

Carbyne is modelled as a linear carbon chain disposed along the $\mathrm{z}$ direction, with $\sim$ $10 \AA$ of vacuum along the non-periodic $x$ and $y$ directions in order to have a proper onedimensional system. The Brillouin zone is sampled with a uniform mesh of $1 \times 1 \times 1000$, $1 \times 1 \times 600$ and $1 \times 1 \times 400$ k-points with the PBE, PBE0 and CAMB3LYP xc functionals respectively in the $1 x 1 x 2$ supercell, with a Fermi-Dirac smearing of $2 \mathrm{mHa}$ (see Sec. S1 of the SI).The unit cell has been relaxed in order to obtain the equilibrium lattice parameter for each exchange-correlation functional, obtaining $2.552 \AA, 2.534$ Aand 2.526 A with the PBE, PBE0 and CAMB3LYP xc functionals respectively.

The input dynamical matrices for the SSCHA utility [6, 7, 8, 9, are computed with the Quantum ESPRESSO [1, 2] code, with a k-point sampling of $1 \times 1 \times 50$ and a Fermi-Dirac smearing of $10 \mathrm{mRy}$, in order to assure definite positive initial Hessian matrices. The force engine of the SSCHA utility is the CRYSTAL [3, 4, code: forces and energies are computed on supercells with 20 atoms per unit cell, containing the unstable phonon mode at the border of the Brillouin zone of the the $1 \times 1 \times 1$ unit cell and assuring converged anharmonic spectra in the thermodynamical limit (see Sec. S3.1 in the SI).

In the SSCHA, the number of configurations on which we calculate the forces depends on the temperature, on the size of the supercell and on the starting guess used. With 20 atoms per unit cell the number of DFT forces needed to converge the free energy is of the order of 1500, while approximately 3000 forces are needed to converge the free energy Hessian.

Optical properties of the distorted phase are computed within the GW approximation using the Yambo [10, 11] code within the plasmon-pole approximation [12]. Eigenvalues and wavefunctions are initially computed with the PBE xc correlation functional and Quantum Espresso. We then performed self-consistent $G W$ calculations on eigenvalues only (ev $G W$ ) for both the Green's function $G$ and the screened electron-electron interaction $W$ in the Plasmon-Pole approximation (PPA). Notice that in general, in self-consistent GW, also the wave-function should be updated, but in Ref. 13] it has been shown that for many systems DFT wave-functions are already quite good and a self-consistency on eigenvalues only can be sufficient.

Excitonic effects for the freestanding carbon chain are then evaluated by solving the Bethe-Salpeter [14, 15] equation (BSE) on top of the self-consistent $G W$ band structure, taking into account processes between the two valence bands and the first four conduction bands. The number of k-points has been fixed to 400 in order to converge the first excitonic peak in the absorption spectrum. Finally, we want to specify that results for the excitons are obtained via a dynamical screening calculated using QP eigenvalues for the BSE equation. Our estimation of the exciton binding energy is in agreement with previous theoretical calculations using the Bethe-Salpeter method for different kinds of polymers [16, 17]. 


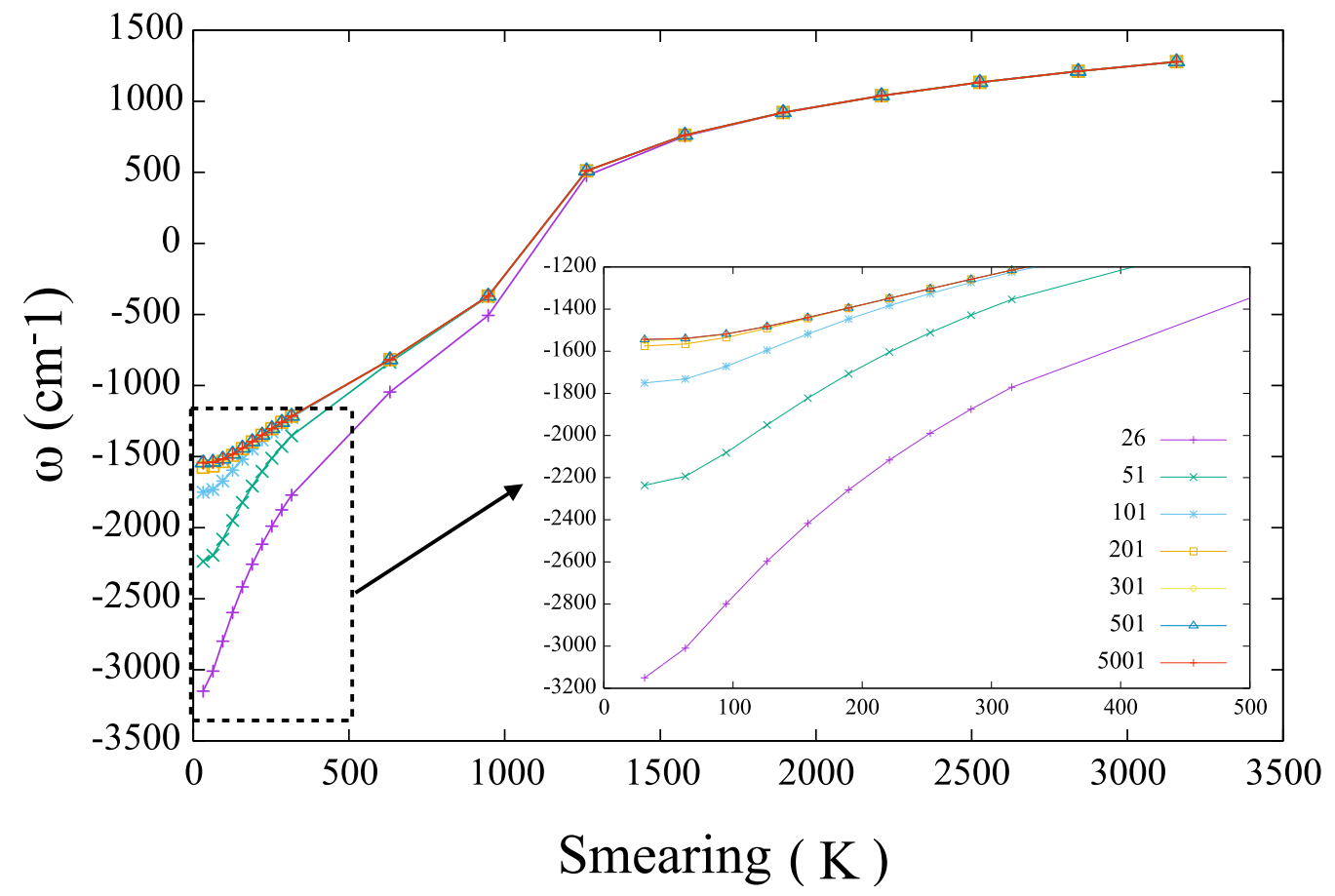

Figure 1: Convergence of the longitudinal phonon mode as a function of the electronic temperature (Smearing) for the PBE exchange-correlation functional for different values of k-points in the irreducible Brillouin zone (IBZ). In the inset we show a zoom around the converged region, highlighted with a black dashed box. 


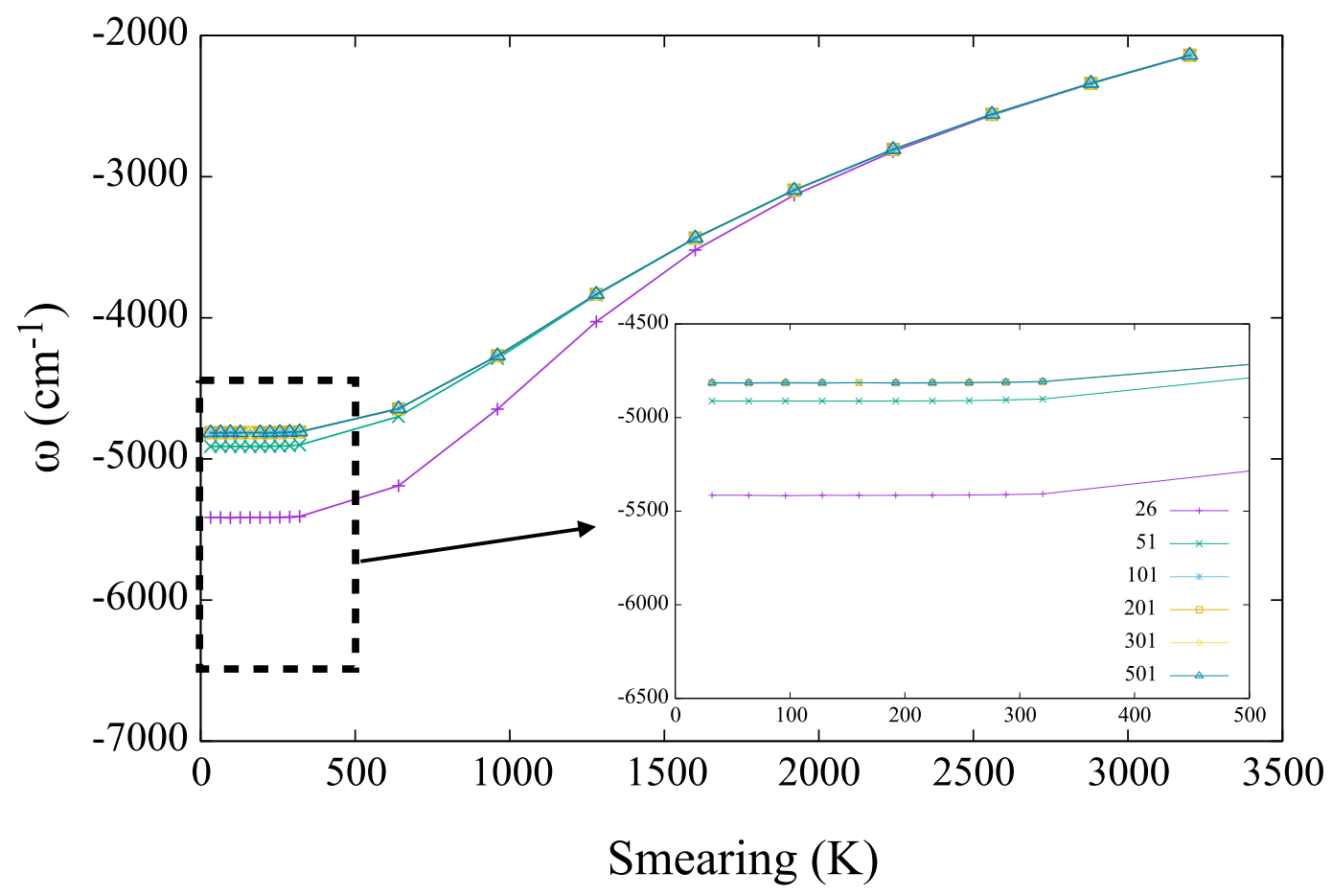

Figure 2: Convergence of the longitudinal phonon mode as a function of the electronic temperature (Smearing) for the PBE0 exchange-correlation functional for different values of k-points in the irreducible Brillouin zone (IBZ). In the inset we show a zoom around the converged region, highlighted with a black dashed box.

\section{Vibrational Properties}

\subsection{Different contributions to the temperature dependent phonon frequency}

Within the SSCHA, the temperature dependent phonons are obtained from the dynamical matrix

$$
\mathcal{D}=\left.\boldsymbol{M}^{-\frac{1}{2}} \frac{\partial^{2} F}{\partial \mathcal{R} \partial \mathcal{R}}\right|_{\mathcal{R}_{e q}} M^{-\frac{1}{2}}
$$

where $\boldsymbol{M}$ is the matrix of the ionic masses $M_{a}$ with $M_{a b}=\delta_{a b} M_{a}$, and $\left.\frac{\partial^{2} F}{\partial \mathcal{R} \partial \mathcal{R}}\right|_{\mathcal{R}_{e q}}$ is the free energy Hessian in the equilibrium configuration. It is

$$
\mathcal{D}=\stackrel{2}{\mathcal{D}}+\mathcal{D}^{\text {bubble }}+\mathcal{D}^{\text {bubble }} \text { beyond }
$$


where

$$
\begin{aligned}
\stackrel{2}{\mathcal{D}} & =\boldsymbol{M}^{-\frac{1}{2}} \mathbf{\Phi} \boldsymbol{M}^{-\frac{1}{2}} \\
\mathcal{D}^{\text {bubble }} & =\boldsymbol{M}^{-\frac{1}{2}} \stackrel{(3)}{\mathbf{\Phi}} \boldsymbol{\Lambda}(0) \stackrel{(3)}{\boldsymbol{\Phi}} \boldsymbol{M}^{-\frac{1}{2}} \\
\mathcal{D}_{\text {beyond }}^{\text {bubble }} & =\boldsymbol{M}^{-\frac{1}{2}} \stackrel{(3)}{\mathbf{\Phi}} \boldsymbol{\Lambda}(0) \boldsymbol{\Theta} \boldsymbol{\Lambda}(0) \stackrel{(3)}{\boldsymbol{\Phi}} \boldsymbol{M}^{-\frac{1}{2}}
\end{aligned}
$$

Analogously, with $\stackrel{0}{\mathcal{D}}$ we refer to the harmonic dynamical matrix:

$$
\stackrel{0}{\mathcal{D}}=\left.\boldsymbol{M}^{-\frac{1}{2}} \frac{\partial^{2} V}{\partial \boldsymbol{R} \partial \boldsymbol{R}}\right|_{\boldsymbol{R}_{0}} \boldsymbol{M}^{-\frac{1}{2}}
$$

where $\left.\frac{\partial^{2} V}{\partial \boldsymbol{R} \partial \boldsymbol{R}}\right|_{\boldsymbol{R}_{0}}$ is the Born-Oppenheimer potential energy Hessian in the 'classical' equilibrium configuration $\boldsymbol{R}_{0}$. The function $\boldsymbol{\Lambda}(0)$ is mainly determined by the eigenvectors and eigenvalues of $\stackrel{2}{\mathcal{D}}$ and the tensor $\boldsymbol{\Theta}$ is the solution of the equation $\boldsymbol{\Theta}=[\mathbb{1}-\stackrel{(4)}{\boldsymbol{\Phi}} \boldsymbol{\Lambda}]^{-1} \stackrel{(4)}{\boldsymbol{\Phi}}$ (See Ref. [7 for more details). In the main text (Fig. 1b) we have included both the bubble contribution to the dynamical matrix, $\mathcal{D}^{\text {bubble }}$, and the contribution of $\mathcal{D}^{\text {beyond }}$ bubble , even if there is no substantial difference for the longitudinal phonon mode. In Fig. 3 we show, for a supercell with 20 atoms in the unit cell, the difference between the anharmonic phonon dispersion with (black dashed) and without (red solid) the $\mathcal{D}$ busbond berm at several temperatures $(\mathrm{T}=$ $0,200,800$ and $1200 \mathrm{~K}$ ) using the PBE0 xc functional.

Moreover, in the framework of the self-consisent harmonic approximation, using suitable ansatz as described in Refs. [7, 18, it is possible to obtain an approximate expression of the one-phonon Green's function $\boldsymbol{G}(\boldsymbol{q}, z)\left(z=\omega+i 0^{+}\right)$:

$$
\boldsymbol{G}^{-1}(\boldsymbol{q}, z)=z^{2} \mathbb{1}-\boldsymbol{M}^{-\frac{1}{2}} \boldsymbol{\Phi} \boldsymbol{M}^{-\frac{1}{2}}-\boldsymbol{\Pi}(\boldsymbol{q}, z)
$$

where $\boldsymbol{\Pi}(\boldsymbol{q}, z)$ is the phonon self-energy, which within the SSCHA can be approximated by the bubble self-energy:

$$
\boldsymbol{\Pi}(\boldsymbol{q}, z) \approx \boldsymbol{\Pi}^{(B)}(\boldsymbol{q}, z)=\boldsymbol{M}^{-\frac{1}{2}} \stackrel{(3)}{\mathbf{\Phi}} \boldsymbol{\Lambda}(\boldsymbol{q}, z) \stackrel{(3)}{\mathbf{\Phi}} \boldsymbol{M}^{-\frac{1}{2}}
$$

From the one-phonon Green's function we obtain the corresponding spectral function $-2 \operatorname{Tr}\left\{\operatorname{Im}\left\{G\left(\boldsymbol{q}, \omega+i 0^{+}\right)\right\}\right\}$. Peaks in this quantity, as a function of $\omega$, signal the presence of collective vibrational excitations (phonons) having certain energies, as they can be probed with inelastic scattering experiments. Multiplying the spectral function by the factor $\omega / 2 \pi$ we obtain the phonon scattering cross-section:

$$
\boldsymbol{\sigma}(\boldsymbol{q}, \omega)=-\frac{\omega \operatorname{Tr}\left\{\operatorname{Im}\left\{G\left(\boldsymbol{q}, \omega+i 0^{+}\right)\right\}\right\}}{\pi}
$$

In order to confirm that the bubble term is converged on a $1 \times 1 \times 20$ supercell, we have adopted the interpolation method for the bubble explained in Ref. [19, 20]. This method is based on the consideration that anharmonic terms are supposed to be short ranged with respect to the harmonic contribution. As shown in Fig. 4. at $\mathrm{T}=0 \mathrm{~K}$ the value of the 
longitudinal phonon mode calculated with this interpolation scheme on larger and larger supercells converges to the same results as the $1 \times 1 \times 20$ (non interpolated) one for both $\mathrm{xc}$ functionals.

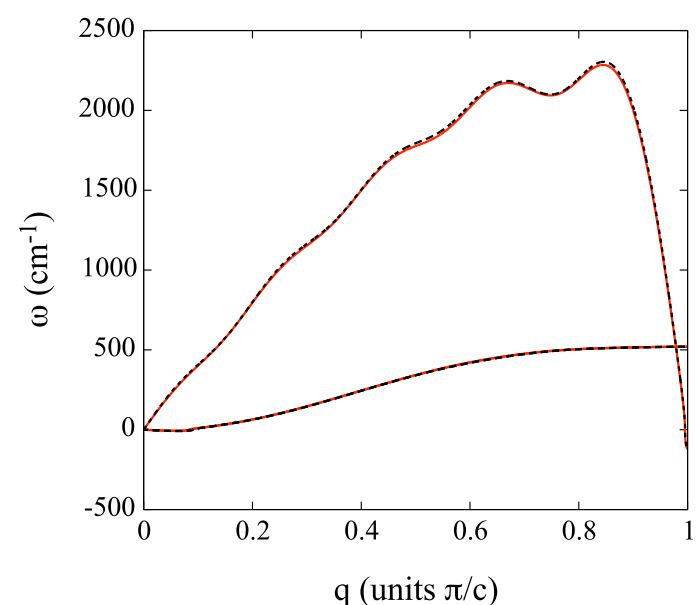

(a)

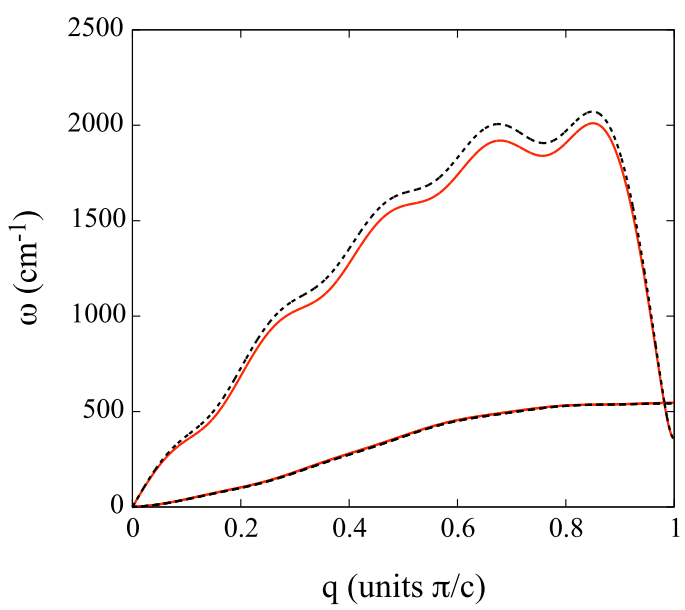

(c)

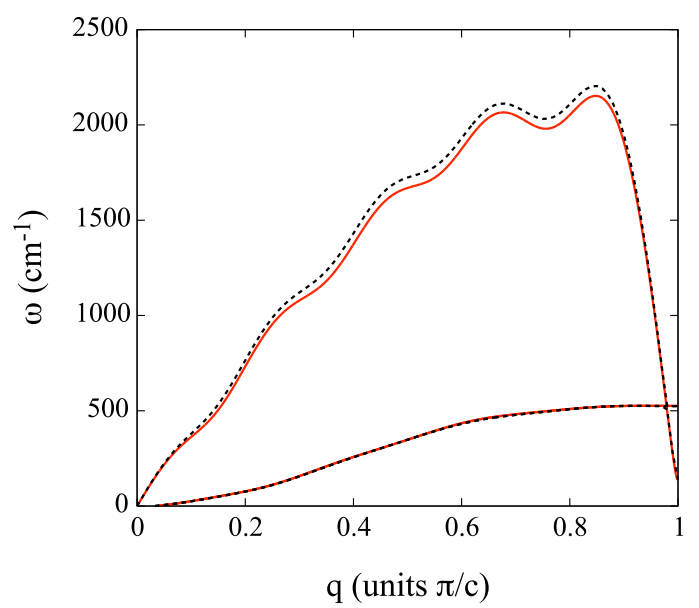

(b)

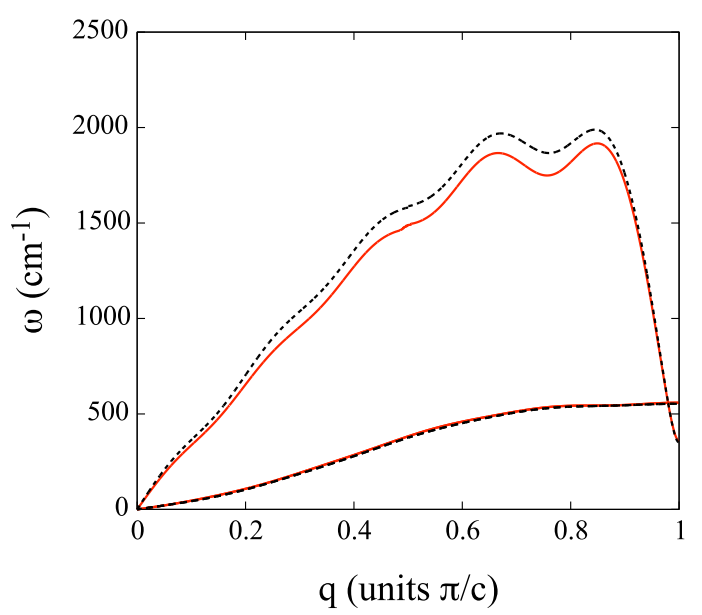

(d)

Figure 3: Comparison of the anharmonic phonon dispersion of cumulene for a supercell with 20 atoms in the unit cell with (black dashed) and without (red solid) the $\mathcal{D}$ beyond buble term at several temperatures: a) $\mathrm{T}=0 \mathrm{~K}, \mathrm{~b}$ ) $\mathrm{T}=200 \mathrm{~K}, \mathrm{c}$ ) $\mathrm{T}=800 \mathrm{~K}$ and d) $\mathrm{T}=1200 \mathrm{~K}$, using the PBE0 xc functional. 


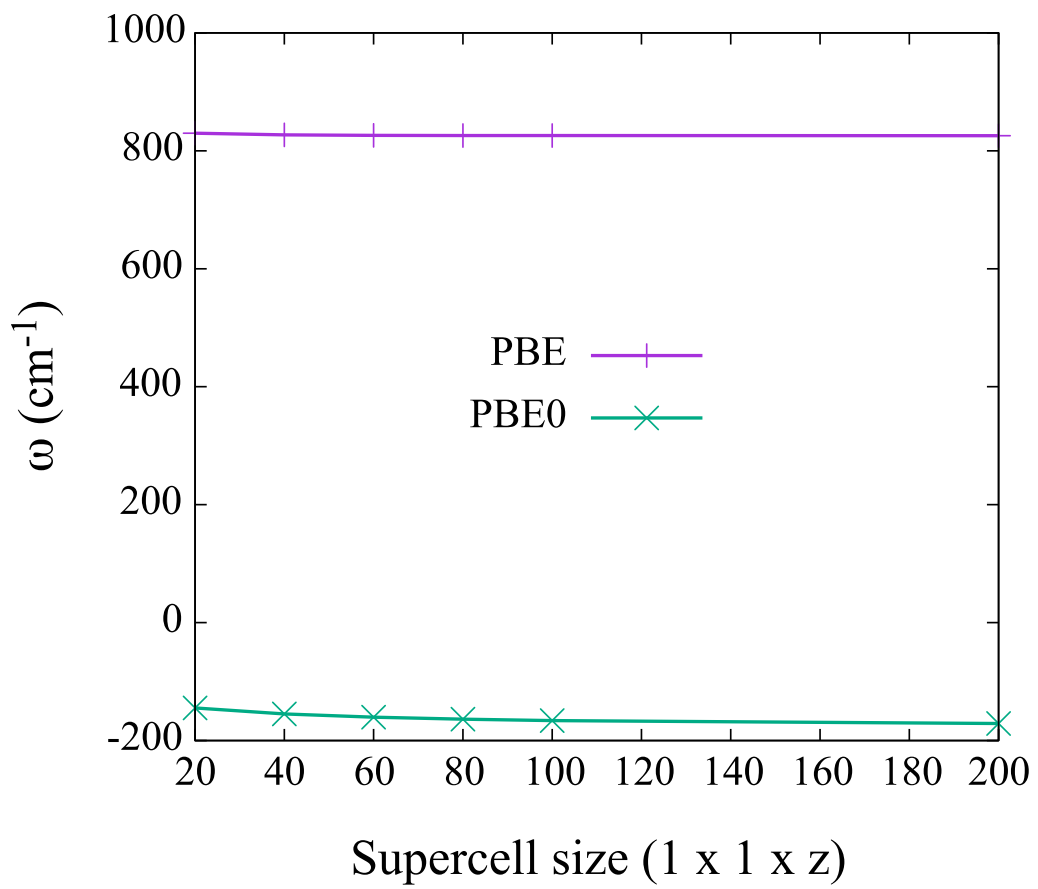

Figure 4: Convergence of the bubble contribution to the dynamical matrix ( $\mathcal{D}^{\text {bubble }}$ ) in the thermodynamic limit as a function of the supercell size. 


\subsection{Static anharmonic phonon dispersion of carbyne}

In Fig. 5 we plot the static phonon dispersion relation of the undistorted metallic phase, i.e. cumulene, as a function of themperature computed with the PBE0 xc functional and taking into account quantum anharmonic effects.

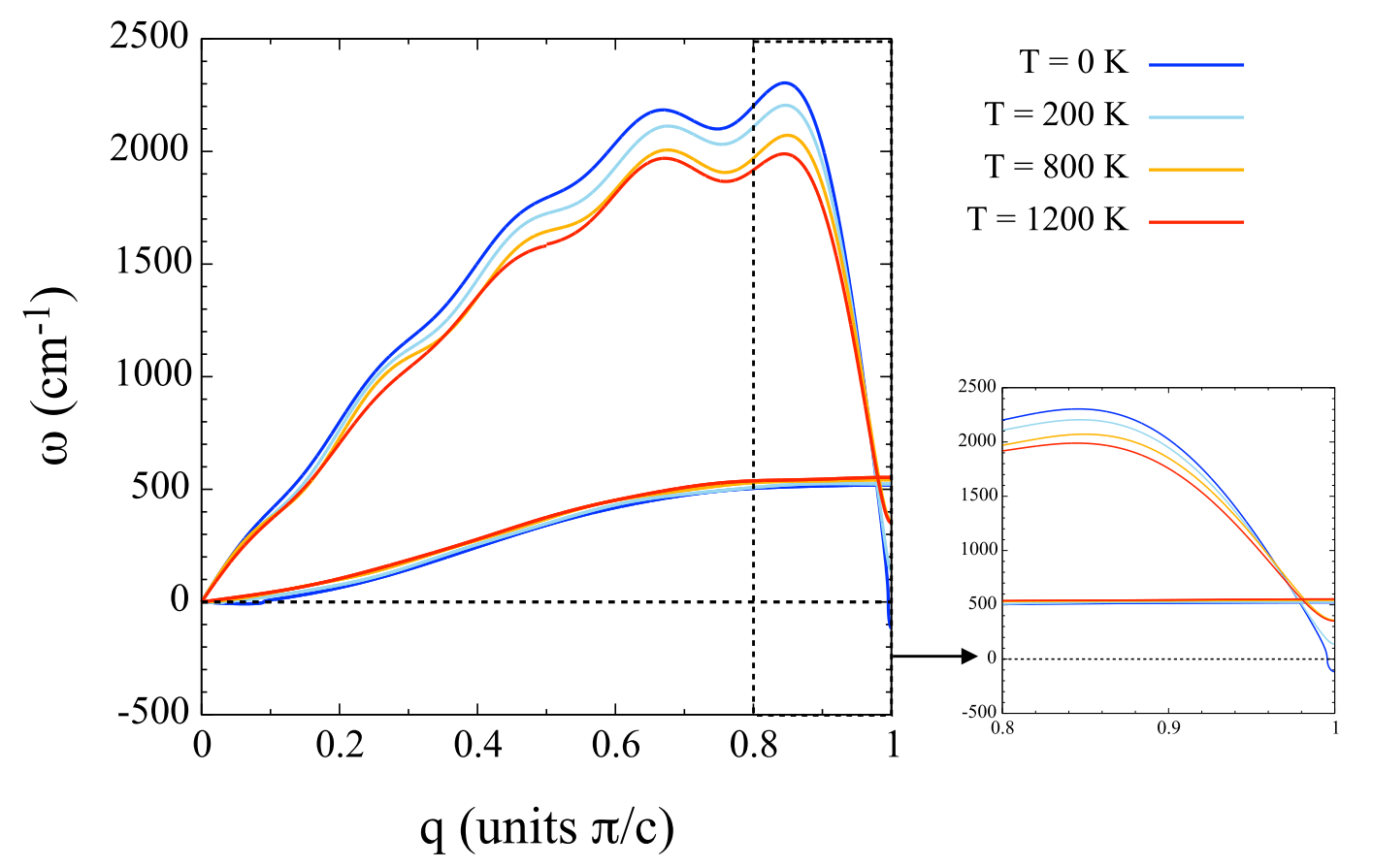

Figure 5: Cumulene static anharmonic phonon dispersion curves at $\mathrm{T}=0 \mathrm{~K}$ (black), $\mathrm{T}=$ $200 \mathrm{~K}$ (red), $\mathrm{T}=800 \mathrm{~K}$ (blue), $\mathrm{T}=1200 \mathrm{~K}$ (magenta) and $\mathrm{T}=1800 \mathrm{~K}$ (green), computed with the PB0 xc functional.

In Fig. 6 we plot the static phonon dispersion relation of the distorted insulating phase, i.e. polyyne, as a function of themperature computed with the PBE0 xc functional and taking into account quantum anharmonic effects. Since polyyne has a unit cell with two atoms per cell, the longitudinal phonon mode responsible for the Peierls distortion, which was at the border of the Brillouin zone in the case of cumulene, is now folded at the center of the Brillouin zone.

Starting from $T=0 \mathrm{~K}$, as we increase temperature the frequency of the longitudinal phonon mode is gradually reduced. Nevertheless, such phonon mode remains always positive (i.e. real) meaning that the insulating phase is always dynamically stable at least up to $T=1800 \mathrm{~K}$. 


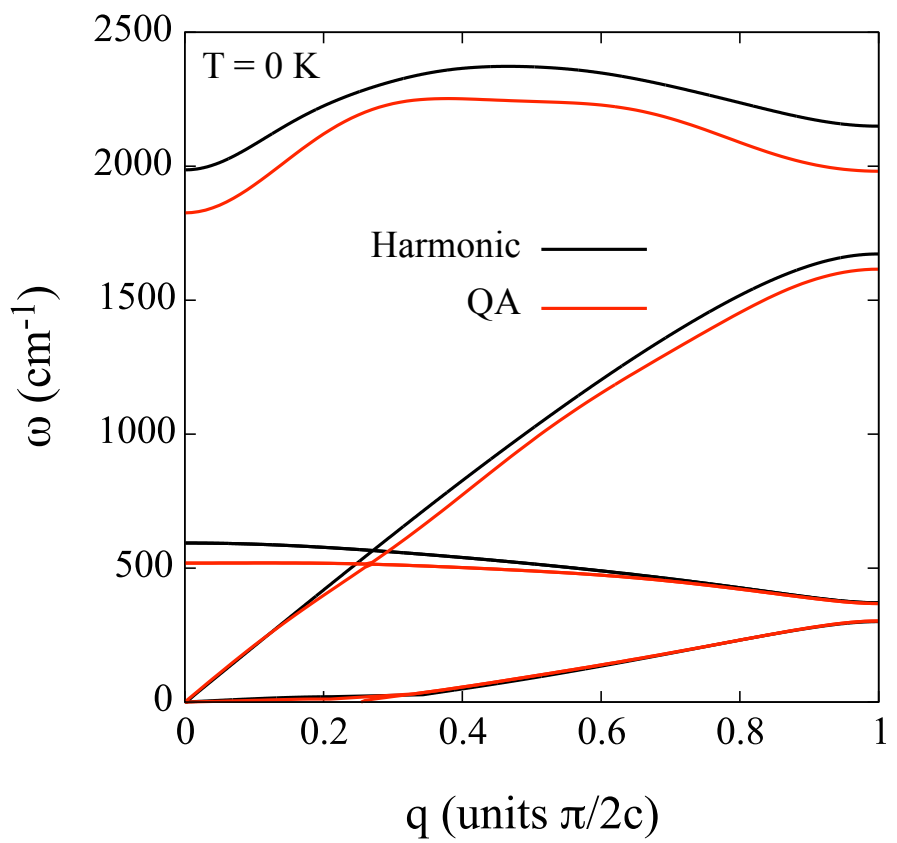

(a)

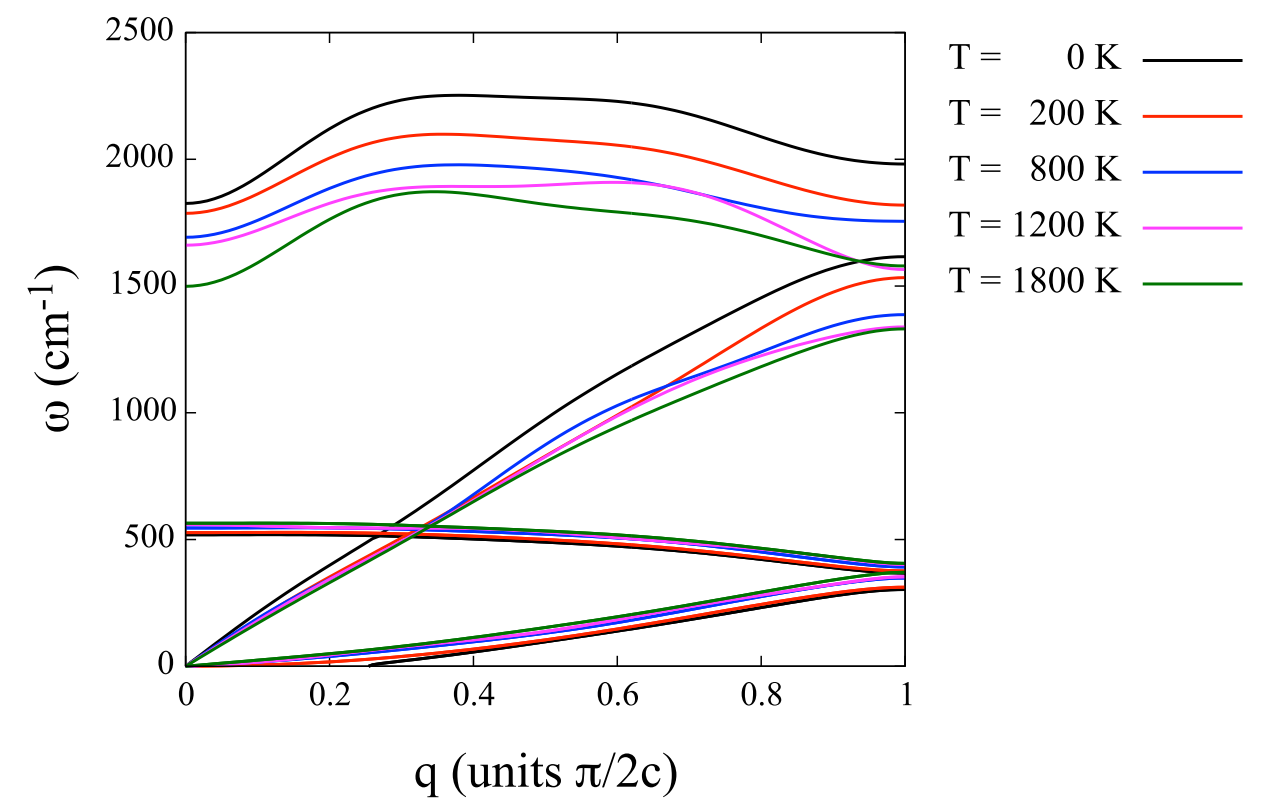

(b)

Figure 6: (a) Harmonic and static anharmonic phonon dispersion relation (obtained by diagonalizing the Hessian of the quantum anharmonic free energy divided by the mass of carbon) of polyyne at $\mathrm{T}=0 \mathrm{~K}$ with PBE0 xc functional.(b) Polyyne static anharmonic phonon dispersion curves at $\mathrm{T}=0 \mathrm{~K}$ (black), $\mathrm{T}=200 \mathrm{~K}$ (red), $\mathrm{T}=800 \mathrm{~K}$ (blue), $\mathrm{T}=$ $1200 \mathrm{~K}$ (magenta) and $\mathrm{T}=1800 \mathrm{~K}$ (green), computed with the PB0 xc functional. 


\subsection{Dynamical anharmonic phonon dispersion of carbyne}

In order to provide theoretical phonon spectra that can be compared with experiments, in Fig. 7 and Fig. 8 we have plotted the dynamic anharmonic phonon dispersion relations of cumulene and polyyne respectively (as described in Sec. 2.1) obtained with the PBE0 xc functional together with quantum anharmonic interactions. We can see that the frequency dependence of the phonon self-energy modifies the high-frequency part of the spectrum in both cases and it also affects the slope of the acoustic modes (i.e. the sound velocity) in polyyne. Such effects are more and more pronounced as temperature is increased. No satellites due to many-body interactions are present.

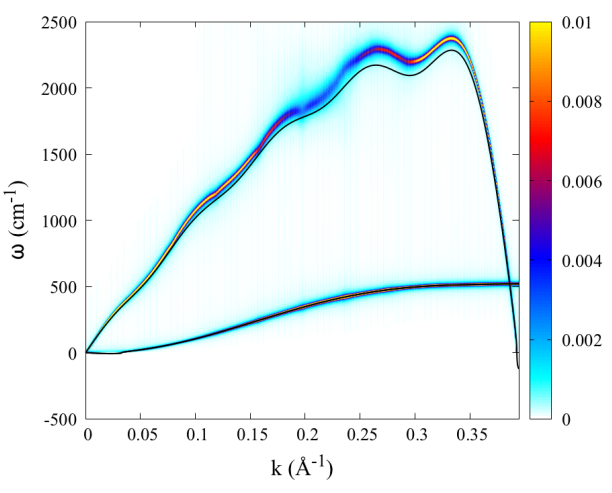

(a)

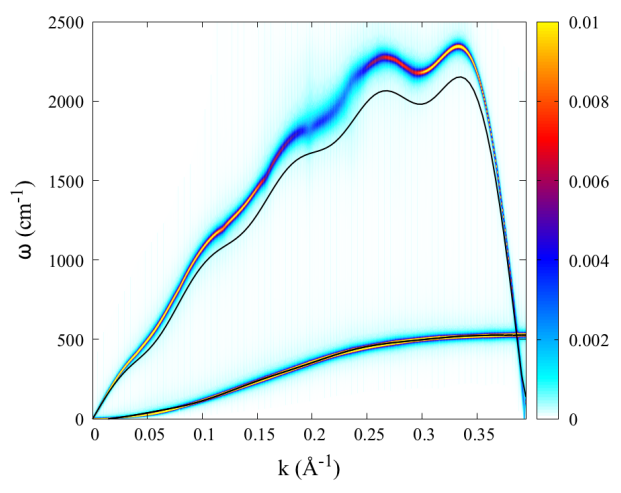

(b)

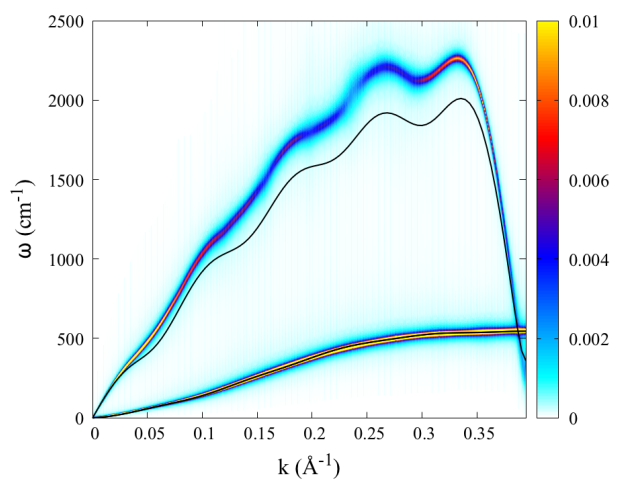

(c)

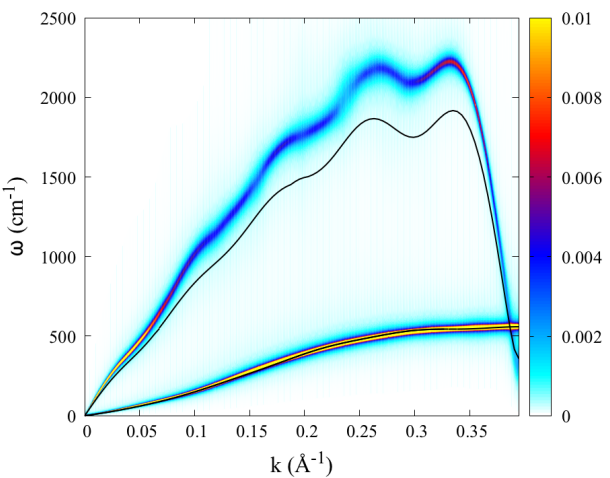

(d)

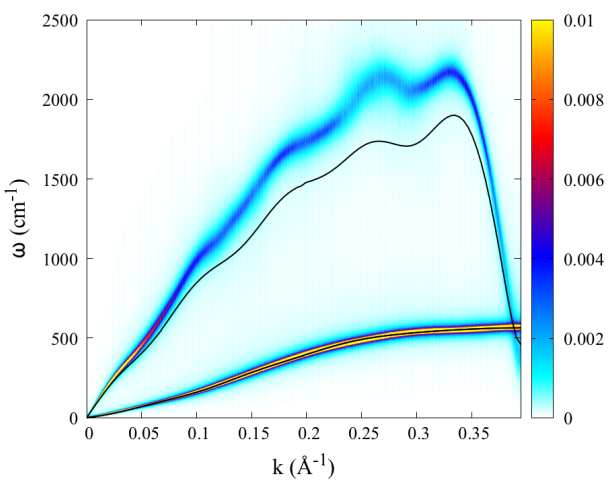

(e)

Figure 7: Cumulene spectral function at a) $\mathrm{T}=0 \mathrm{~K}$, b) $\mathrm{T}=200 \mathrm{~K}$, c) $\mathrm{T}=800 \mathrm{~K}$, d) $\mathrm{T}=$ $1200 \mathrm{~K}$ and e) $\mathrm{T}=1800 \mathrm{~K}$, computed with the PB0 xc functional. Solid black lines denote the static anharmonic phonon dispersion curves. The color code is determined by the value of the cross section $\sigma(\boldsymbol{q}, \omega)$ defined in Eq. 7 . 


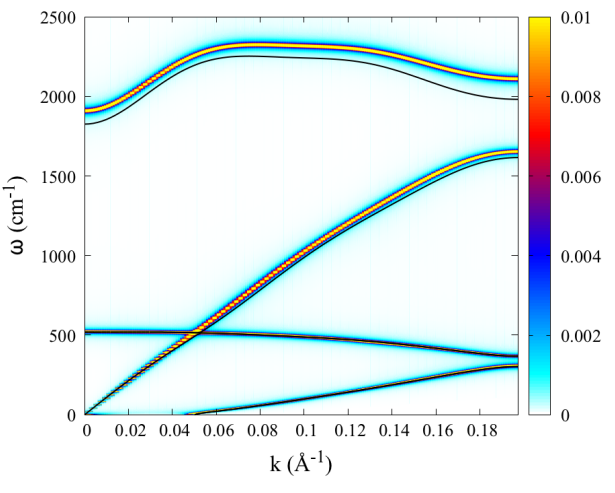

(a)

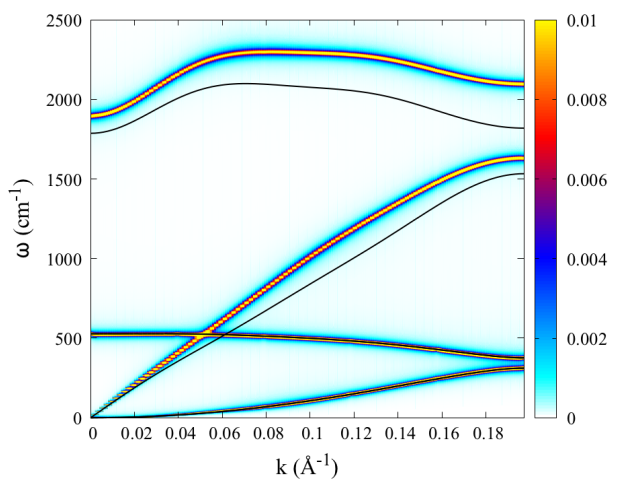

(b)

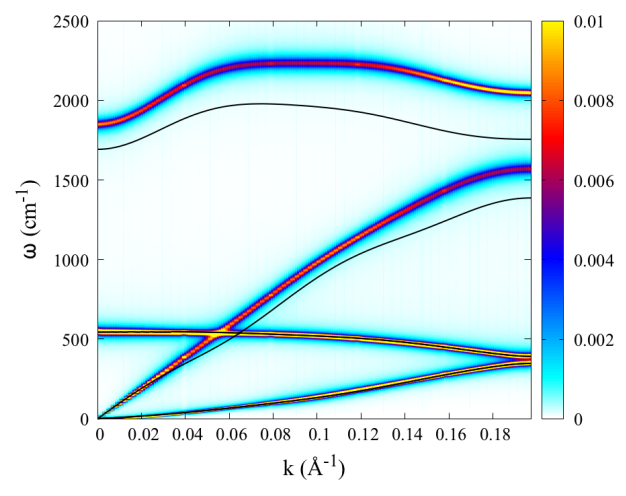

(c)

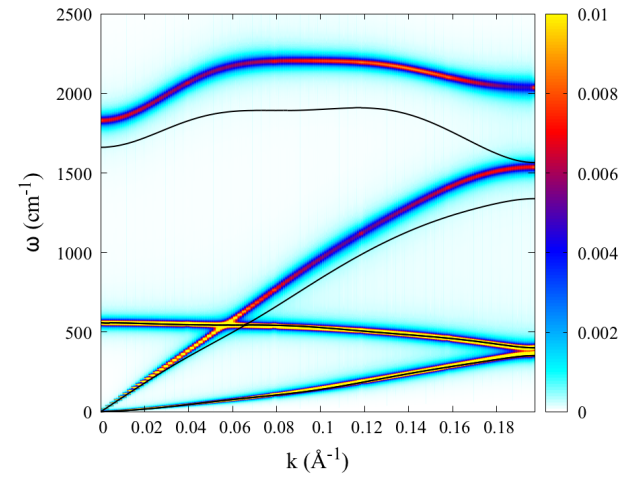

(d)

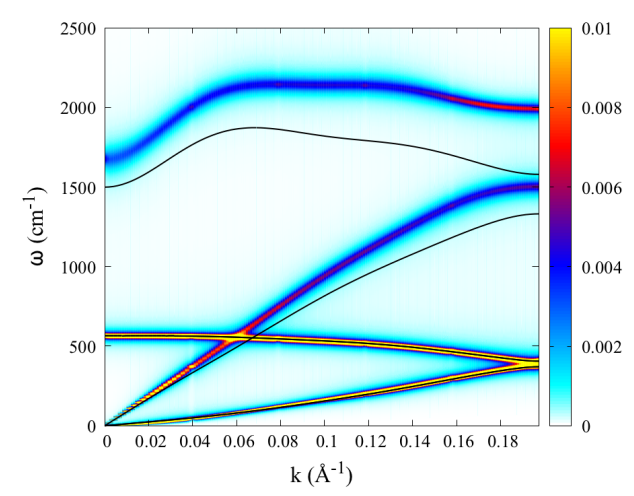

(e)

Figure 8: Polyyne spectral function at a) $\mathrm{T}=0 \mathrm{~K}, \mathrm{~b}) \mathrm{T}=200 \mathrm{~K}$, c) $\mathrm{T}=800 \mathrm{~K}, \mathrm{~d}$ ) $\mathrm{T}=$ $1200 \mathrm{~K}$ and e) $\mathrm{T}=1800 \mathrm{~K}$, computed with the PB0 xc functional. Solid black lines denote the static anharmonic phonon dispersion curves. The color code is determined by the value of the cross section $\sigma(\boldsymbol{q}, \omega)$ defined in Eq. 7 . 


\subsection{Temperature stability of the metallic phase}

In Fig. 9 we have plotted the squared static anharmonic longitudinal phonon frequency of cumulene $\left(\omega_{L O}\right)$, taking also into account its sign. Assuming a mean-field picture, where $\omega_{L O} \propto \sqrt{1-T / T_{C}}$, we linearly interpolated $\omega^{2}$ and we estimated that the metallic phase becomes a metastable phase (i.e. $\omega_{L O}>0$ ) for $T \gtrsim 75 \mathrm{~K}$.

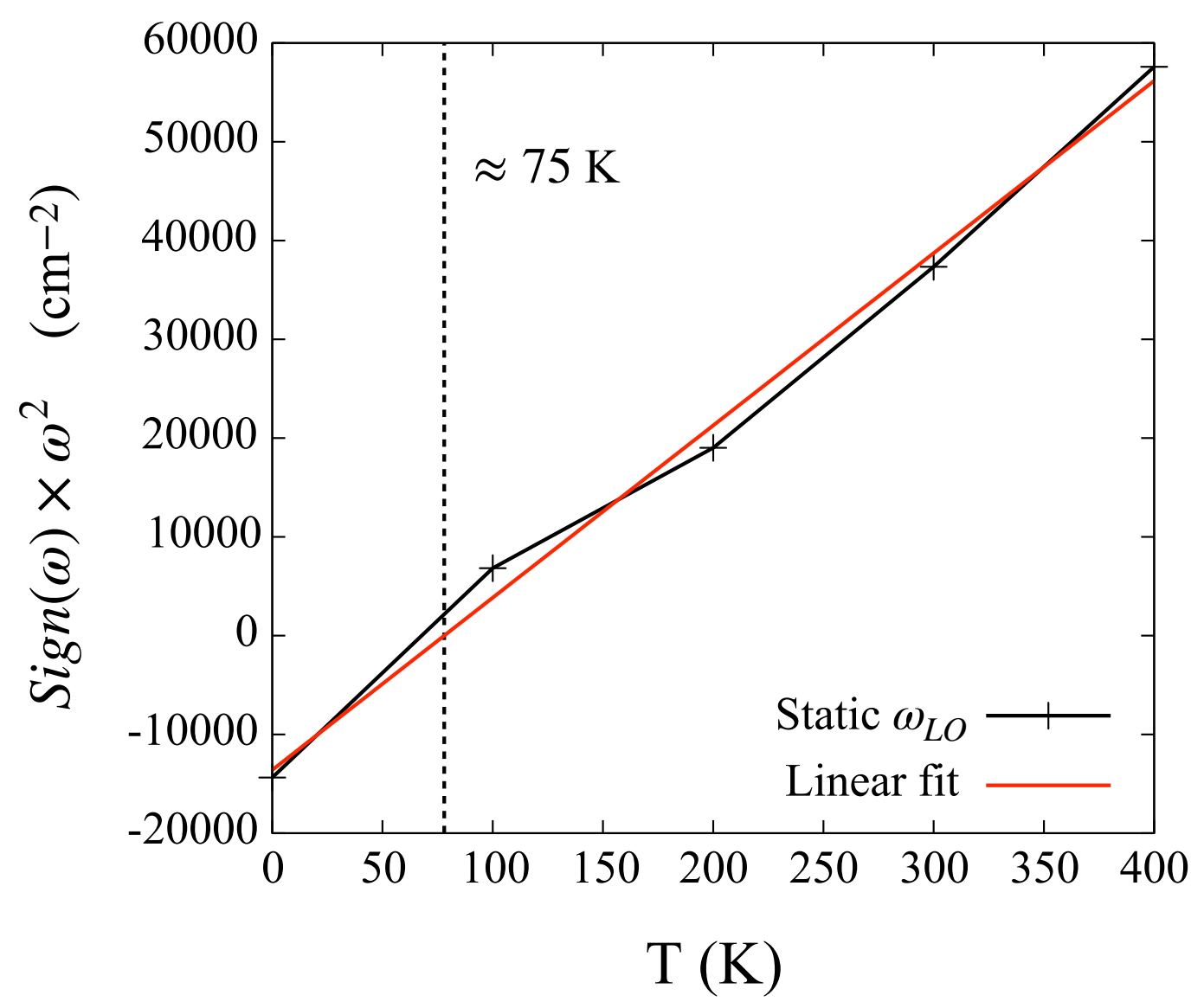

Figure 9: Squared static anharmonic longitudinal phonon frequency of cumulene $\omega_{L O}^{2}$, taking also into account its sign $\operatorname{Sign}(\omega)$ (black line). The solid red line represents a linear fit of the data. 


\subsection{Extrapolation of the charge density wave critical temperature from free energy difference}

In Fig. 10 we have plotted the free energy difference $(\Delta F)$ between the distorted and undistored minima as a function of temperature obtained with the PBE0 xc functional and with quantum anharmonic interactions. Such quantity decreases as temperature is increased and by linearly interpolating $\Delta F$ at high temperatures we extrapolated $T_{c} \approx 3300 \mathrm{~K}$.

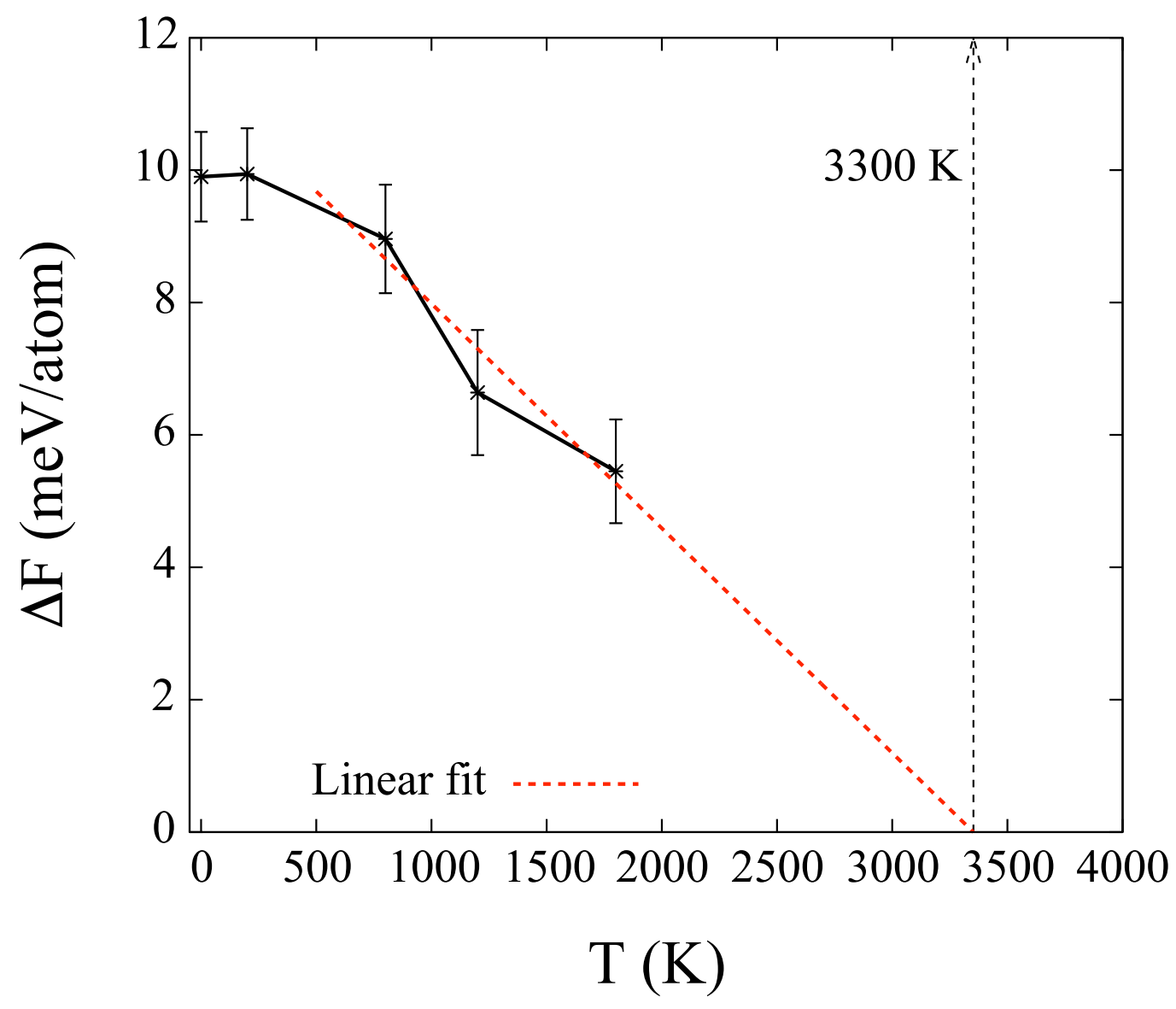

Figure 10: Free energy difference $(\Delta F)$ between the undistorted (cumulene) and distorted (polyyne) minima as a function of temperature computed with the PBE0 xc functional and taking into account quantum anharmoncitiy. The red dashed line represent a linear fit of the high-temperature points in order to extrapolate the charge density wave critical temperature $\left.T_{C D W} \approx 3300 \mathrm{~K}\right)$. 


\subsection{Quantum anharmonic relaxation of polyyne's lattice parameter}

We performed lattice realxation of polyyne taking into account quantum anharmonicity in order to have a better comparison with the experimental temperature evolution $(T \in[40$ : $300] \mathrm{K}$ ) of the longitudinal Raman-active mode $\omega_{L O}$ (see Fig. S19(d) in the suplemental information of Ref [21]). To do so we computed the total free energy (i.e. Born-Oppenheimer static energy + anharmonic vibrational free energy) of the distorted phase as a function of the lattice parameter at the two closest temperatures we analyzed in the main text (i.e. $T=0 \mathrm{~K}$ and $T=200 \mathrm{~K}$ ). For both temperatures and for each value of the lattice parameter we performed a full SSCHA relaxation of internal lattice coordinates. Then we employed a fourth-order polynomial to fit the free energy points (Fig. 2.6). The equilibrium lattice parameter is then the minimum of such polynomial. Results are summarized in Tab. 1 .

\begin{tabular}{c|c|c|c}
\hline $\mathrm{T}(K)$ & $2 \mathrm{c}(\AA)$ & BLA $(\AA)$ & $\omega_{L O}\left(\mathrm{~cm}^{-1}\right)$ \\
\hline \multicolumn{4}{c}{ Harmonic lattice parameter } \\
\hline 0 & 2.534 & 0.0969 & 1909.3 \\
200 & 2.534 & 0.0990 & 1894.5 \\
\hline \multicolumn{4}{c}{ Quantum anharmonic lattice parameter } \\
\hline 0 & 2.541 & 0.0980 & 1880.7 \\
200 & 2.535 & 0.0981 & 1879.2 \\
\hline
\end{tabular}

Table 1: Polyyne's BLA and dynamic longitudinal optical phonon mode $\left(\omega_{L O}\right)$ as a function of temperature $T$ computed using both the harmonic and the relaxed quantum anharmonic lattice parameters $(2 c)$.

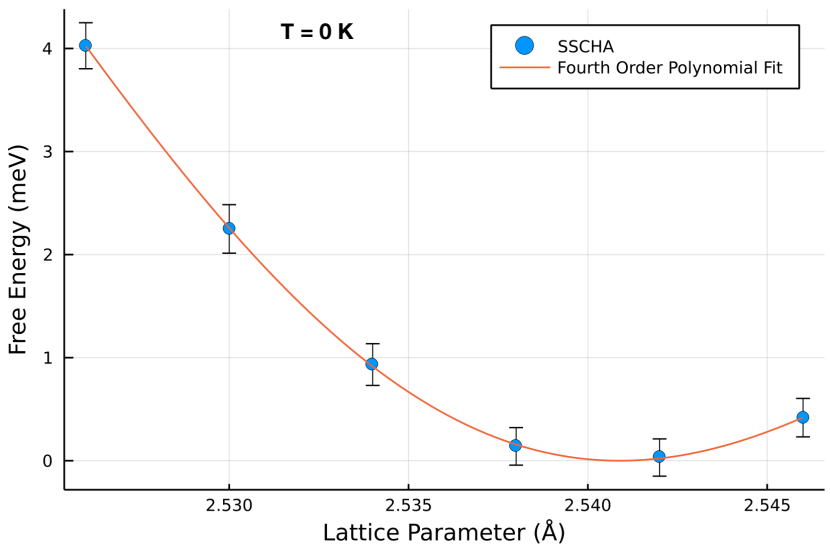

(a)

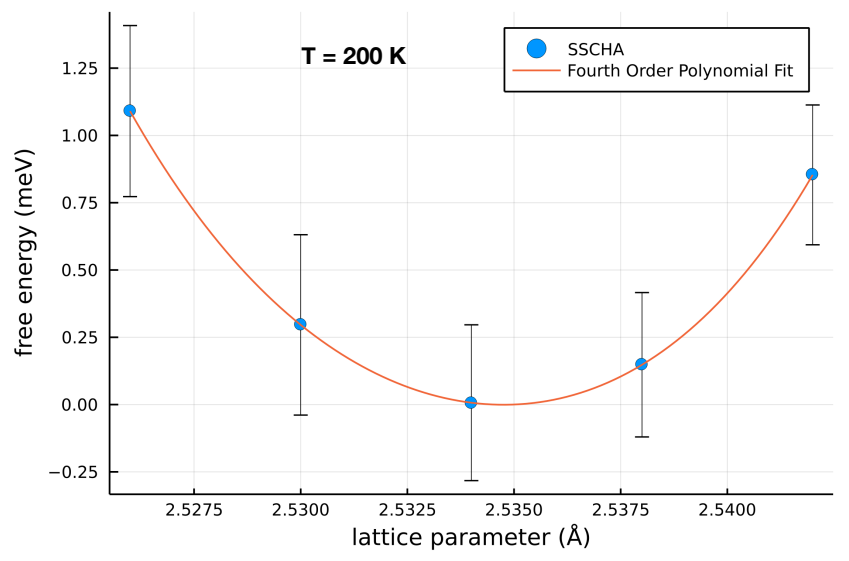

(b)

Figure 11: Total anharmonic free energy of polyyne (i.e. Born-Oppenheimer static energy + anharmonic vibrational free energy) as a function of lattice parameter for different temperatures $T$. The curve is shifted in such a way that the minimum of the polynomial fit has a 0 total free energy. 


\section{Electronic Properties}

\subsection{Self-consistent $G W$ electronic bandstructure of polyyne at the PBE0 equilibrium geometry}

In Fig. 12 we show the convergence on the number of self-consistent GW cycles: it is possible to observe that at the third cycle (i.e. $G_{2} W_{2}$ ) the corrections on eigenvalues has converged.

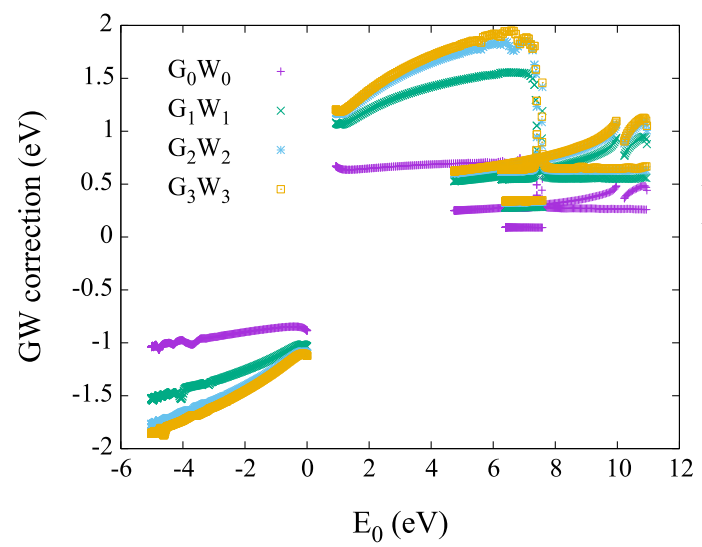

(a)

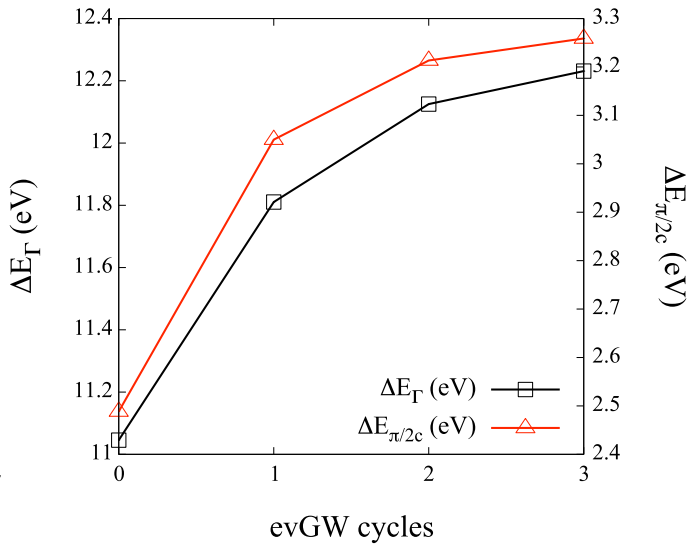

(b)

Figure 12: Convergence on the number of self-consistent GW cycles. (a) $G W$ correction on electronic eigenvalues versus DFT eigenvalues; (b) $G W$ correction on the quasiparticle gap at the center $\left(\Delta E_{\Gamma}\right)$ and at the border $\left(\Delta E_{\pi / 2 c}\right)$ of the Brillouin zone.

In Fig. 13 we compare the electronic band structure of the distorted insulating phase polyyne at the PBE0 equilibrium geometry obtained via different approximations: DFTPBE (black line), DFT-PBE0 (blue line), $\mathrm{G}_{0} \mathrm{~W}_{0}+$ plasmon-pole approximation (red line) and $\mathrm{G}_{0} \mathrm{~W}_{0}+$ full frequency approach to the correlation self-energy (green line). It is possible to observe that the inclusion of nonlocal electron-electron correlations opens up the quasiparticle band-gap from $0.93 \mathrm{eV}$ (PBE) to $2.03 \mathrm{eV}$ (PBE0). Moreover, comparing the electronic dispersion relation obtained via hybrid functional with that coming from manybody perturbation theory (i.e. $\mathrm{G}_{0} \mathrm{~W}_{0}$ ) we can see that we commit an error of just 0.4 $\mathrm{eV}$, since at the $\mathrm{G}_{0} \mathrm{~W}_{0}$ we have an energy gap $\sim 2.4 \mathrm{eV}$. Finally, we also verified that the plasmon-pole approximation for the dynamic correlation electronic self-energy is justified by performing a full frequency calculation: the quasiparticle gap obtained differs only by $\sim 0.2 \mathrm{eV}$.

The convergence parameters are set to:

- 50 Ry for the number of $\boldsymbol{G}$-vectors in the exchange self-energy;

- 40 for the number of bands in the polarization function;

- 10 Ry for number of $\boldsymbol{G}$-vectors block in the dielectric constant;

- 150 bands for the expansion of the Green's function; 


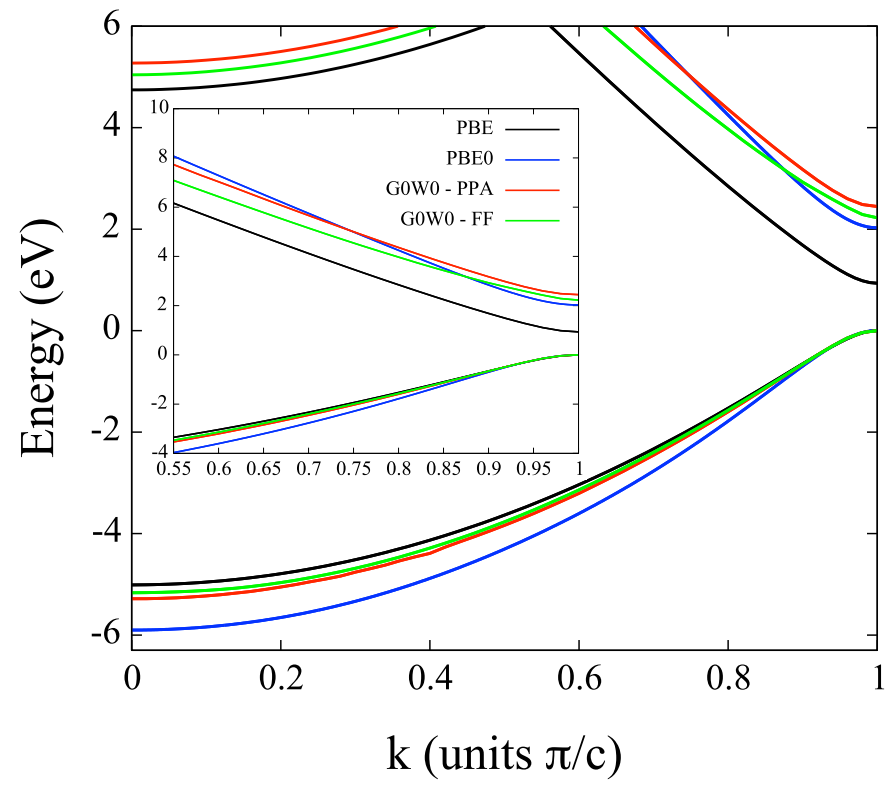

Figure 13: Comparison of the electronic dispersion relation of the distorted phase (polyyne) with different techniques computed at the PBE0 equilibrium geometry: DFT-PBE (black line), DFT-PBE0 (blue line), $\mathrm{G}_{0} \mathrm{~W}_{0}+$ plasmon-pole approximation (red line) and $\mathrm{G}_{0} \mathrm{~W}_{0}+$ full frequency approach to the correlation self-energy (green line). In the inset we show a zoom around the quasi-particle gap at the border of the Brillouin zone. 


\section{CAMB3LYP analysis of carbyne}

It was recently suggested that the CAMB3LYP hybrid exchange-correlation functional 22 ] would give an order parameter (i.e. the BLA) closer to the more accurate diffusion Monte Carlo (DMC) results 23. In order to probe the reliability of such xc functional for carbyne, we have performed the same analysis done in the main text with the PBE0 hybrid functional.

The CAMB3LYP lattice parameter we obtained corresponds to $2.526 \AA$, which is $\sim 2 \%$ smaller with respect to the DMC one $(2.5187 \AA$ [23]). However the BLA obtained with CAMB3LYP, $0.131 \AA$, is just $\sim 4 \%$ smaller with respect to the DMC one, $0.136 \AA[23]$ ), while that obtained with the PBE0 hybrid functional, $0.131 \stackrel{A}{A}$, is $\sim 30 \%$ smaller with respect to the DMC one.

In Fig. 14 we have plotted third order polynomials fits (solid lines) of the total energies close to the distorted phase (dots) obtained via diffusion quantum Monte Carlo [23] (DMC, black), RPA 24] (blue), DFT-PBE0 (red) and DFT-CAMB3LYP (orange) hybrid exchangecorrelation functionals at their respective equilibrium geometries. The curvature of the total energy is proportional to the phonon frequency. While PBE0 underestimates the frequency of the longitudinal phonon mode with respect to DMC, CAMB3LYP slightly overestimates it. Moreover, the curvature of the PBE0 total energy is practically coincident with that computed through RPA 24], confirming the reliability of the PBE0 hybrid functional.

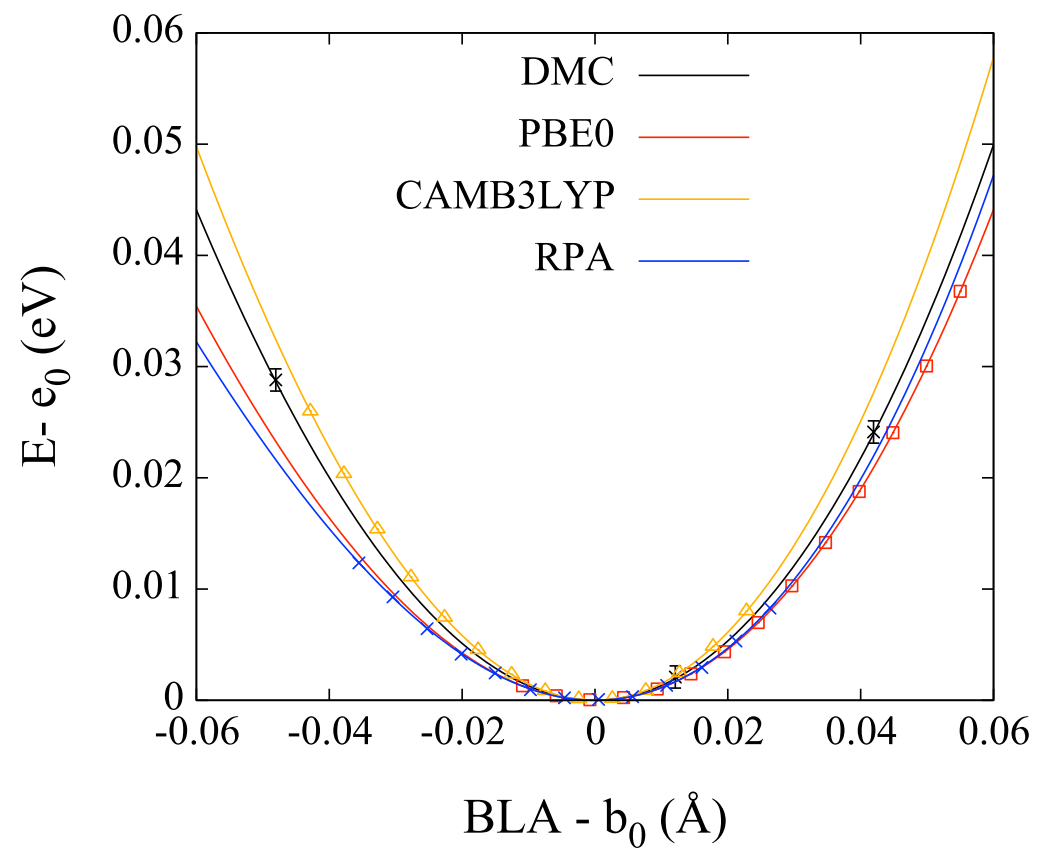

Figure 14: Comparison of the total energy curvature close to the distorted insulating minimum (i.e. polyyne) obtained via diffusion quantum Monte Carlo [23] (DMC, black), RPA 24] (blue), DFT-PBE0 (red) and DFT-CAMB3LYP (orange) hybrid exchangecorrelation functionals. Solid lines represent third order polynomials fit of available data. Here $b_{0}$ is the equilibrium BLA, while $e_{0}$ is the value of the total energy at the equilibrium geometry. 
The static DFT value of the longitudinal phonon mode obtained using the CAMB3LYP functional is $2277.22 \mathrm{~cm}^{-1}$, which is $\sim 9 \%$ bigger with respect to the DMC value 2084 $\mathrm{cm}^{-1}$ 23. On the other hand the PBE0 longitudinal phonon mode has a frequency 1987 $\mathrm{cm}^{-1}$, which is $\sim 1 \%$ smaller with respect to the RPA value $2000 \mathrm{~cm}^{-1}[24$ and $\sim 5 \%$ smaller with respect to DMC.

In Fig. 15 and Fig. 16 we provide the static and the dynamic phonon dispersion relations of the distorted phase obtained with the CAMB3LYP hybrid functional at its equilibrium geometry and taking into account quantum anharmonic contributions. Similar considerations to those already discussed in the case of the PBE0 xc functional apply here: there are no substantial differences from a qualitative point of view.

\begin{tabular}{c|c|c}
\hline $\mathrm{T}(K)$ & BLA $(\AA)$ & $\omega_{L O}\left(\mathrm{~cm}^{-1}\right)$ \\
\hline 0 & 0.1348 & 2226.5 \\
300 & 0.1384 & 2245.2 \\
800 & 0.1433 & 2197.6 \\
\hline
\end{tabular}

Table 2: Polyyne's BLA and dynamic longitudinal optical phonon mode $\left(\omega_{L O}\right)$ as a function of temperature $T$ computed via the CAMB3LYP hybrid functional.

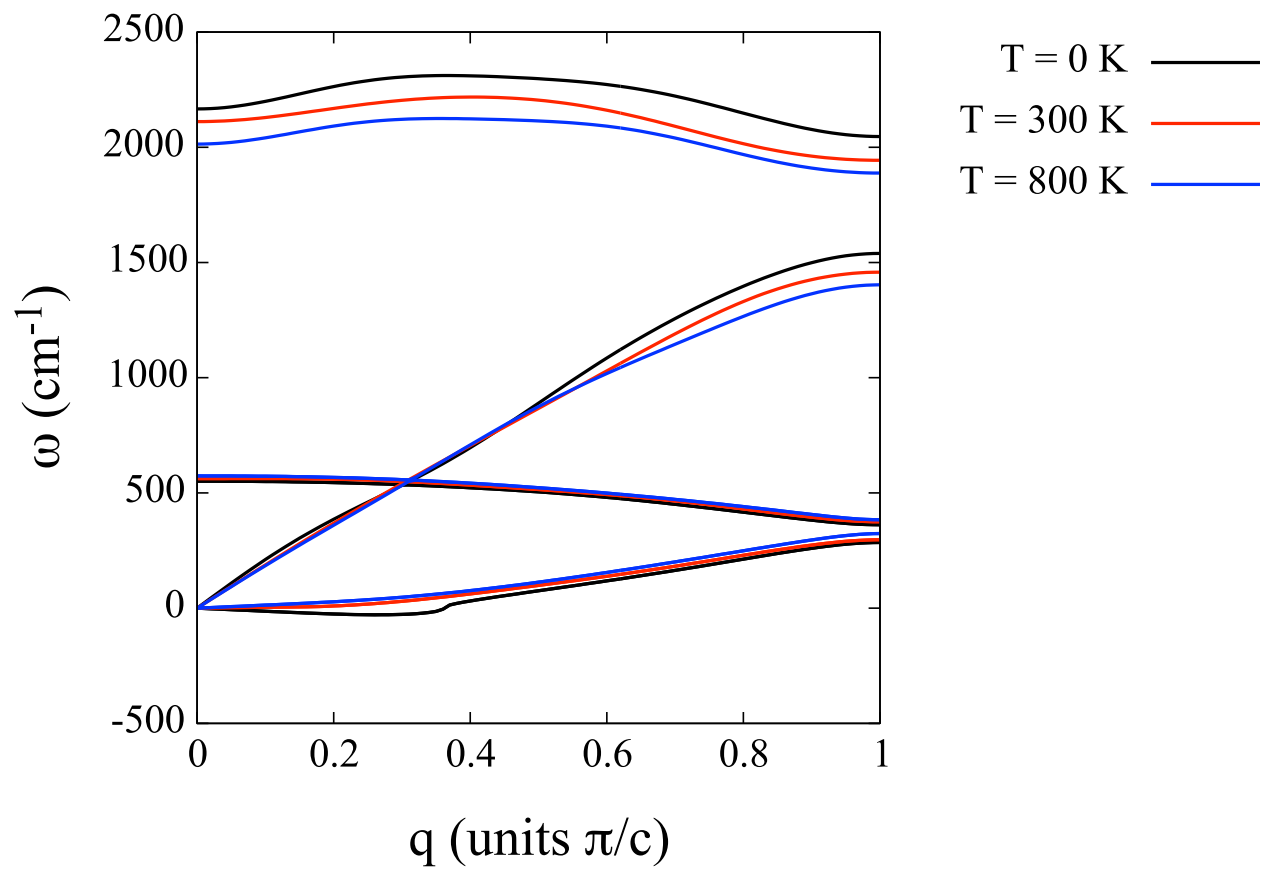

Figure 15: Polyyne static anharmonic phonon dispersion curves at $\mathrm{T}=0 \mathrm{~K}$ (black), $\mathrm{T}=$ $300 \mathrm{~K}$ (red) and $\mathrm{T}=800 \mathrm{~K}$ (blue), computed with the CAMB3LYP xc functional. 


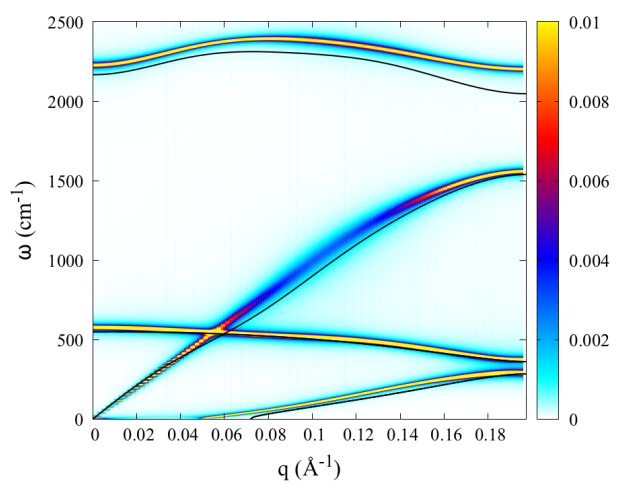

(a)

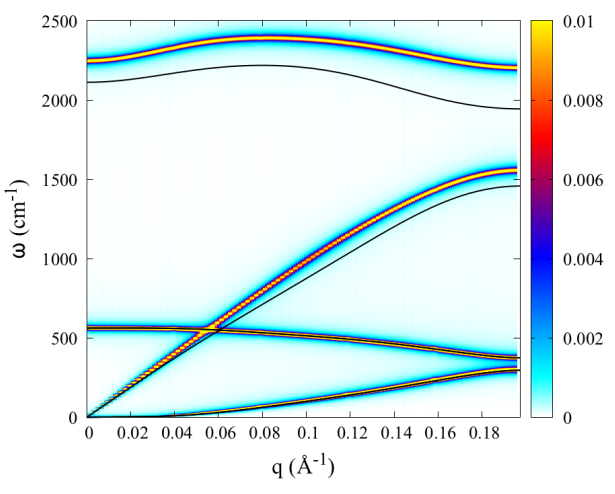

(b)

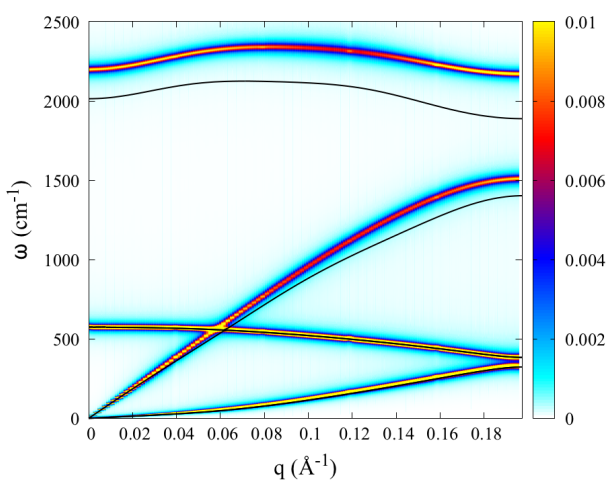

(c)

Figure 16: Polyyne spectral function at a) $\mathrm{T}=0 \mathrm{~K}, \mathrm{~b}) \mathrm{T}=300 \mathrm{~K}$ and c) $\mathrm{T}=800 \mathrm{~K}$, computed with the CAMB3LYP xc functional. Solid black lines denote the static anharmonic phonon dispersion curves. The color code is determined by the value of the cross section $\sigma(\boldsymbol{q}, \omega)$ defined in Eq. 7

Concerning the optics, in Fig. 17 we compare the electronic band structure of the distorted insulating phase polyyne at the CAMB3LYP equilibrium geometry obtained via different approximations: DFT-PBE (black line), DFT-CAMB3LYP (orange line), $\mathrm{G}_{0} \mathrm{~W}_{0}$ + plasmon-pole approximation (red line) and $\mathrm{G}_{0} \mathrm{~W}_{0}+$ full frequency approach to the correlation self-energy (blue line). It is possible to observe that the inclusion of nonlocal electron-electron correlations opens up the quasiparticle band-gap from $1.98 \mathrm{eV}$ (PBE) to $4.39 \mathrm{eV}$ (CAMB3LYP) which is $\sim 22 \%$ bigger with respect to the DMC value, $3.6 \mathrm{eV} 23$. Moreover, comparing the electronic dispersion relation obtained via hybrid functional with that coming from many-body perturbation theory (i.e. $\mathrm{G}_{0} \mathrm{~W}_{0}$ ) we can see that we commit an error of $47 \%$, since at the $\mathrm{G}_{0} \mathrm{~W}_{0}$ we have an energy gap $\sim 2.99 \mathrm{eV}$ (which is the closest one to the DMC energy gap). 


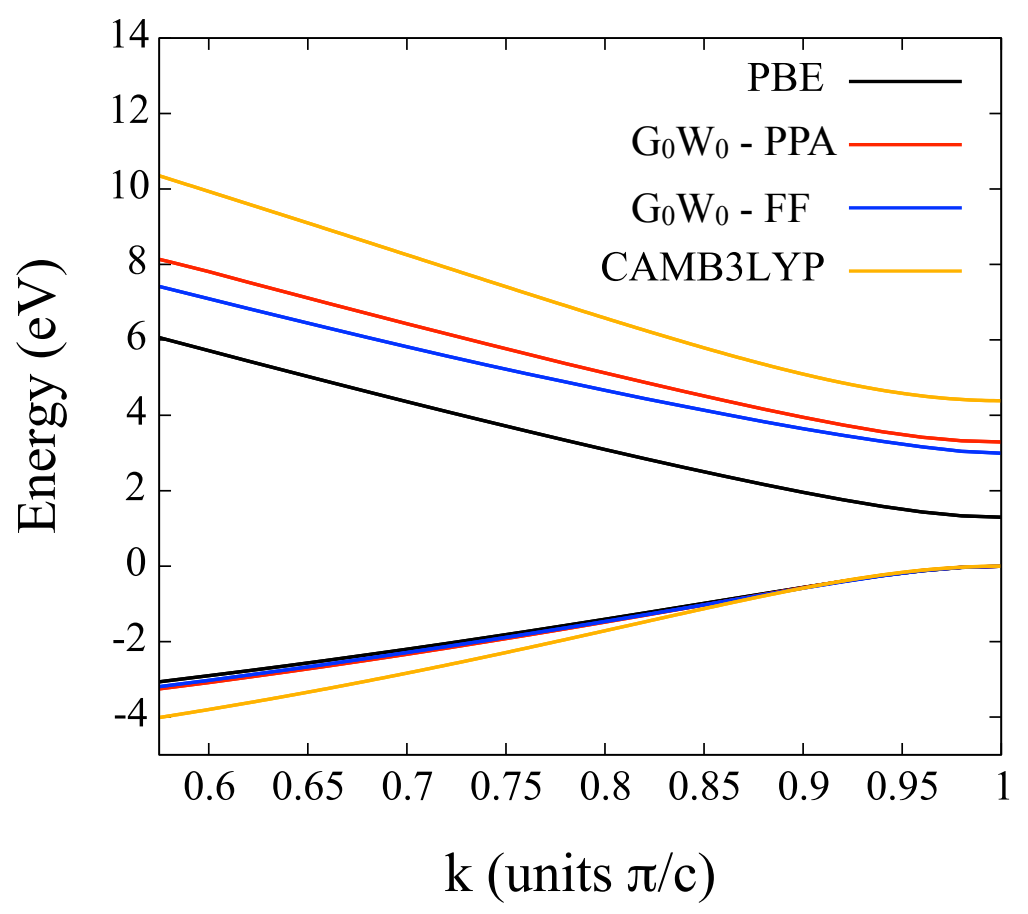

Figure 17: Comparison of the electronic dispersion relation of the distorted phase (polyyne) with different techniques computed at the CAMB3LYP equilibrium geometry: DFT-PBE (black line), $\mathrm{G}_{0} \mathrm{~W}_{0}+$ plasmon-pole approximation (red line), $\mathrm{G}_{0} \mathrm{~W}_{0}+$ full frequency approach to the correlation self-energy (blue line) and DFT-CAMB3LYP (orange line).

On the other hand notice that the PBE0 hybrid functional at the PBE0 equilibrium geometry gives an energy gap $44 \%$ smaller with respect to the DMC value, but only $9 \%$ smaller with respect to the $\mathrm{G}_{0} \mathrm{~W}_{0}$ one (see Sec 3.1 .

Finally, if we adopt the PBE0 hybrid functional on top of the CAMB3LYP equilibrium geometry we obtain an energy gap of $2.58 \mathrm{eV}$, which is only $\sim 13 \%$ smaller with respect to the $\mathrm{G}_{0} \mathrm{~W}_{0}$ one at the same equilibrium geometry. 


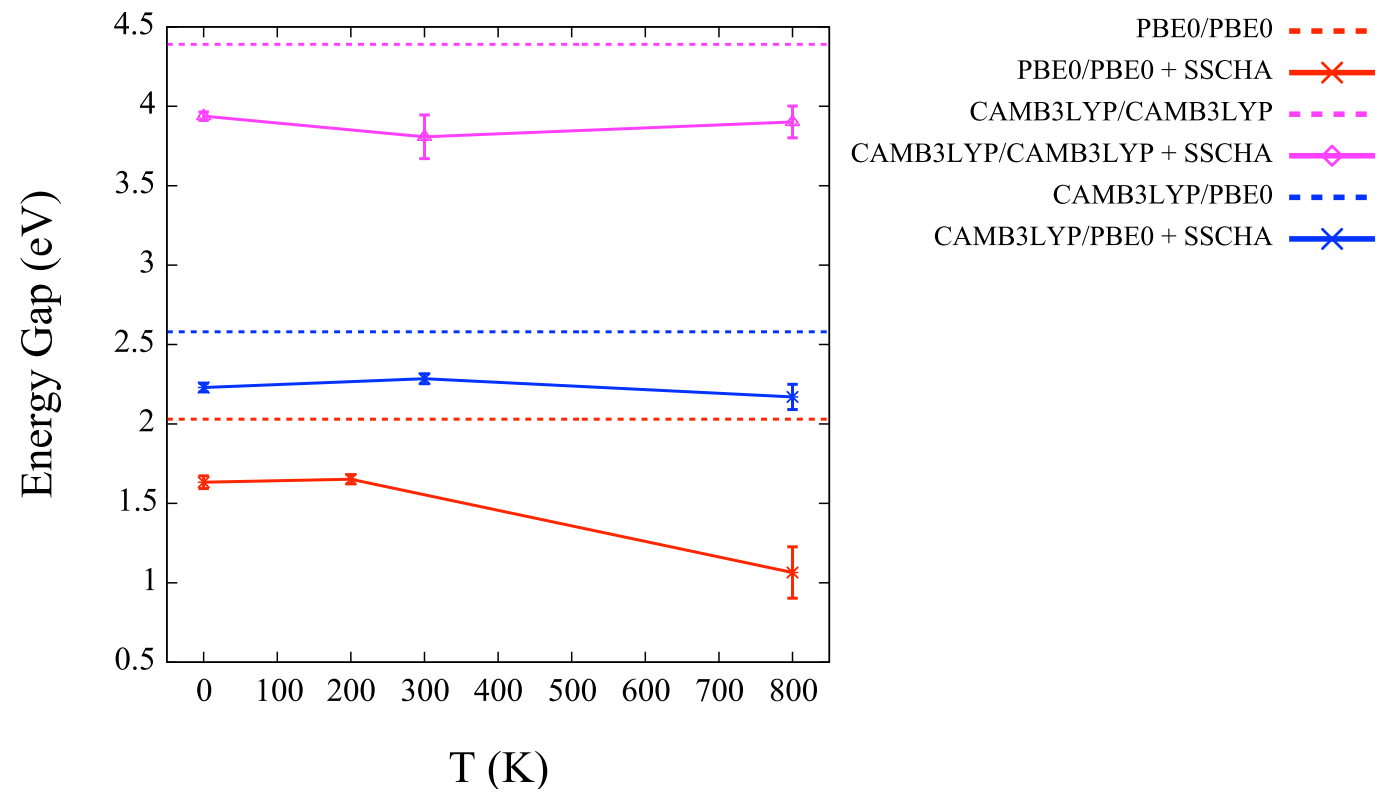

Figure 18: Quasiparticle energy gap as a function of temperature obtained at fixed atom positions at the DFT level (dashed lines) and renormalized by both quantum and thermal atomic fluctuations (solid lines) performed at the PBE0 equilibrium geometry with the PBE0 xc functional (red), CAMB3LYP equilibrium geometry with the CAMB3LYP xc functional (magenta) and CAMB3LYP equilibrium geometry with the PBE0 xc functional (blue).

\section{References}

[1] Giannozzi, P. et al. Quantum espresso: a modular and open-source software project for quantum simulations of materials. Journal of Physics: Condensed Matter 21, 395502 (2009).

[2] Giannozzi, P. et al. Advanced capabilities for material modeling with quantum espresso. Journal of Physics: Condensed Matter 29, 465901 (2017).

[3] Dovesi, R. et al. Crystal14: A program for the ab-initio investigation of crystalline solids. International Journal of Quantum Chemistry 114, 1287-1317 (2014).

[4] Dovesi, R. et al. Quantum-mechanical condensed matter simulations with crystal. WIREs Comput Mol Sci. 8, 1360 (2018).

[5] Oliveira, D. V., Laun, J., Peintinger, M. F. \& Bredow, T. Bsse-correction scheme for consistent gaussian basis sets of double- and triple-zeta valence with polarization quality for solid-state calculations. Journal of Computational Chemistry 40, 2364-2376 (2019).

[6] Errea, I., Calandra, M. \& Mauri, F. Anharmonic free energies and phonon dispersions from the stochastic self-consistent harmonic approximation: Application to platinum and palladium hydrides. Phys. Rev. B 89, 064302 (2014). 
[7] Bianco, R., Errea, I., Paulatto, L., Calandra, M. \& Mauri, F. Second-order structural phase transitions, free energy curvature, and temperature-dependent anharmonic phonons in the self-consistent harmonic approximation: Theory and stochastic implementation. Phys. Rev. B 96, 014111 (2017).

[8] Monacelli, L., Errea, I., Calandra, M. \& Mauri, F. Pressure and stress tensor of complex anharmonic crystals within the stochastic self-consistent harmonic approximation. Phys. Rev. B 98, 024106 (2018).

[9] Monacelli, L. et al. The stochastic self-consistent harmonic approximation: calculating vibrational properties of materials with full quantum and anharmonic effects. J. Phys.: Condens. Matter 33, 363001 (2021).

[10] Marini, A., Hogan, C., Grüning, M. \& Varsano, D. yambo: An ab initio tool for excited state calculations. Computer Physics Communications 8, 180 (2009).

[11] Sangalli, D. et al. Many-body perturbation theory calculations using the yambo code. Journal of Physics: Condensed Matter 31, 325902 (2019).

[12] Rojas, H. N., Godby, R. W. \& Needs, R. J. Space-time method for ab initio calculations of self-energies and dielectric response functions of solids. Phys. Rev. B 74, 10 (1995).

[13] Faber, C., Boulanger, P., Duchemin, I., Attaccalite, C. \& Blase, X. Many-body green's function gw and bethe-salpeter study of the optical excitations in a paradigmatic model dipeptide. J. Chem. Phys. 139, 194308 (2013).

[14] Strinati, G. Application of the green's functions method to the study of the optical properties of semiconductors. Rivista del Nuovo Cimento 11, 12 (1988).

[15] Bussi, G. Effects of the electron-hole interaction on the optical properties of materials: the bethe-salpeter equation. Physica Scripta 2004, 141 (2003).

[16] van der Horst, J.-W., Bobbert, P. A. \& Michels, M. A. J. Calculation of excitonic properties of conjugated polymers using the bethe-salpeter equation. J. Chem. Phys. 114, 6950 (2001).

[17] Pedersen, T. G. Density-functional-based tight-binding calculation of excitons in conjugated polymers. Phys. Rev. B 69, 075207 (2004).

[18] Ribeiro, G. A. S. et al. Strong anharmonicity in the phonon spectra of pbte and snte from first principles. Phys. Rev. B 97, 014306 (2018).

[19] Bianco, R., Errea, I., Calandra, M. \& Mauri, F. High-pressure phase diagram of hydrogen and deuterium sulfides from first principles: Structural and vibrational properties including quantum and anharmonic effects. Phys. Rev. B 97, 214101 (2018).

[20] Zhou, J. S. et al. Anharmonicity and doping melt the charge density wave in single-layer tise $_{2}$. Nano Lett. 7, 4809--4815 (2020).

[21] Shi, L. et al. Confined linear carbon chains as a route to bulk carbyne. Nature Materials 15, 634-639 (2016). 
[22] Yanaia, T., Tewb, D. P. \& Handy, N. C. A new hybrid exchange-correlation functional using the coulomb-attenuating method (cam-b3lyp). Chemical Physics Letters 393, 51-57 (2004).

[23] Mostaani, E., Monserrat, B., Drummond, N. D. \& Lambert, C. J. Quasiparticle and excitonic gaps of one-dimensional carbon chains. Phys. Chem. Chem. Phys. 18, 1481014821 (2016).

[24] Ramberger, B. \& Kresse, G. New insights into the 1d carbon chain through the rpa. Phys. Chem. Chem. Phys. 23, 5254 (2021). 